\title{
THE HOST GALAXIES OF IR LUMINOUS QUASARS
}

\author{
D.L. CLEMENTS \\ Institut d'Astrophysique Spatial \\ Bat 121, Universite Paris XI, \\ F-91405 ORSAY CEDEX, France \\ A.C. BAKER \\ Service d'Astrophysique \\ CEA Saclay, Orme de Merisiers Bat 709, \\ F91191 Gif-sur-Yvette CEDEX, France \\ AND \\ C.J. LIDMAN \\ European Southern Observatory \\ La Silla, Chile
}

A connection is suspected between quasars and ultraluminous IR galaxies (ULIRGs). Almost all ULIRG activity is triggered by mergers (e.g. Clements et al. 1996, MNRAS, 279, 477). We thus investigate the relationship between quasars and ULIRGs by examining the relationship between FIR luminosity and host morphology in quasars. We use the $z<0.3$ imaging survey of PG quasars by McLeod \& Rieke (1994, ApJ, 420, 58; 431, 137 ), and ancillary data, to determine which quasars in the survey have disturbed hosts. This is combined with FIR data from Sanders et al. (1989, $A p J, 347,29)$. We apply Survival Analysis techniques to see if the FIR luminosity of the disturbed and undisturbed host quasars differ. We find, with $99.99 \%$ confidence, that the disturbed host quasars have higher FIR luminosity. We observe a small, but complete, sample of six quasars selected to have $L_{\text {FIR }}>10^{11.8} L_{\odot}$, and a comparison sample of quasars with similar redshifts and B-band absolute magnitudes, but without detected FIR emission. At least 3 of the FIR luminous quasars are found in disturbed hosts while the non-FIR luminous quasars all lie in undisturbed hosts. The FIR luminosity in ULIRGs is triggered by interactions. If ULIRGs evolve into quasars we would expect FIR luminous quasars to be younger, and to share more characteristics with ULIRGs, especially disturbed host morphology. These studies are consistent with this model for quasar evolution. 